\title{
ORIGINAL
}

\section{EVOLUCIÓN DE LA PANDEMIA POR EL VIRUS DE LA GRIPE (H1N1) 2009 EN LA COMUNIDAD AUTÓNOMA DE LA RIOJA}

\author{
Eva María Martínez Ochoa, Carmen Quiñones Rubio, Ma Eugenia Lezaún Larumbe, Ángela \\ Blanco Martínez y Milagros Perucha González
}

Servicio de Epidemiología y Prevención Sanitaria de La Rioja.

\section{RESUMEN}

Fundamento. El objetivo de este estudio ha sido realizar un análisis de la evolución de la pandemia por el virus de la gripe (H1N1) 2009 en la Comunidad Autónoma de La Rioja tanto en atención primaria como en hospitalaria.

Métodos. El periodo de estudio incluyó desde la semana 282009 hasta la 3-2010. La información de los casos de gripe se obtuvo de las bases informatizadas de atención primaria (OMI), de los listados remitidos por los servicios hospitalarios de medicina preventiva, del laboratorio de referencia de La Rioja y de los formularios de notificación individual. Se realizó una descripción de los casos según distribución por semana, sexo, edad, factores de riesgo, evolución y complicaciones. Se utilizó la regresión binaria logística para estimar las odds ratios ajustadas y su intervalo de confianza al $95 \%$, de recibir tratamiento antiviral según factores de riesgo.

Resultados. Hasta la semana epidemiológica 3 de 2010 se declararon a través del sistema OMI de atención primaria 7.096 casos de sospecha de gripe (H1N1) 2009, fueron hospitalizados 111 casos con diagnóstico confirmado por laboratorio. Requirieron atención en cuidados intensivos $5(4,5 \%)$ y 2 fallecieron. Todos ellos presentaban factores de riesgo. Ninguno de los niños ni de las mujeres embarazadas hospitalizados precisaron ingreso en cuidados intensivos.

Conclusiones. Durante la pandemia la gripe (H1N1) 2009 fue una enfermedad propia de la población pediátrica y joven, apareciendo con menor frecuencia en las personas mayores de 60 años. La evolución clínica en La Rioja cursó de forma leve en la mayoría de las personas diagnosticados.

Palabras clave: Brote. Pandemia. Subtipo H1N1 del Virus de la Influenza A. Gripe humana. España. Factores de riesgo. Atención primaria de salud. Hospital.

\section{ABSTRACT \\ Evolution of the Virus Pandemic Flu (H1N1) 2009 in the Autonomous Community of La Rioja}

Background. The aim of this study has been to analyze the development of pandemic influenza (H1N1) 2009 in La Rioja autonomous (cases in primary healthcare and cases hospitalized).

Methods. We have included all cases with 2009 H1N1 influenza from week 28-2009 to 3-2010. The main information was collected through Primary Healthcare databases (OMI), lists of Preventive Medicine Hospital Departments, reference laboratory and individual standardized forms. This information was analyzed and described according to week, sex, age, comorbidities, clinical development and medical complication. We explore the association of to antiviral treatment and risk factors. This association was assessed using odds ratios (OR) and $95 \%$ confidence interval (CI). Adjustment for confounders was performed using unconditional logistic regression to estimate the odds ratio (OR) and $95 \%$ confidence intervals $(\mathrm{CI})$.

Results. From 29-2009 week to 3-2010 week, a total of 7096 pandemic influenza A(H1N1) cases were notified from Primary Healthcare and 111 laboratory confirmed severe cases were admitted to hospital. Five cases were admitted to an ICU $(4,5 \%)$ and 2 cases died. All this patients had comorbidities. None of children and pregnant female were admitted to an ICU.

Conclusions. Children and young person have been population with more cases of pandemic influenza (H1N1) 2009. Clinical evolution of pandemic in the majority cases in La Rioja, has been mild.

Key words: Epidemiology. Sentinel surveillance. Disease Outbreaks. Influenza A virus, H1N1 subtype. Grippe. Influenza, human. Spain. Risk factors Primary health care. Hospital.

Correspondencia:

Eva María Martínez Ochoa

Gran Vía $18,7^{\mathrm{a}}$ planta

26071 Logroño (La Rioja)

eva.martinez@larioja.org 


\section{INTRODUCCIÓN}

A lo largo de los años en el mundo se han presentado diversas pandemias de gripe ${ }^{1}$, apareciendo a intervalos entre diez y cincuenta años. Durante el siglo XX se produjeron tres importante pandemias, la primera en el año $1918^{2}$, más conocida como «Gripe Española», fue la de mayor gravedad causando más de 40 millones de muertes en menos de un año y entre el $25-30 \%$ de la población enferma. En $1957^{1}$ apareció la llamada «Gripe Asiática», que presentó una mortalidad con un patrón similar a las epidemias estacionales, aunque con una afectación mucho mayor de ancianos y niños. En 1968 se produjo la última pandemia del siglo $\mathrm{XX}$, que fue más suave que la de $1957^{3}$. Aunque existieron importantes diferencias entre ellas, todas tenían como característica común su rápida difusión.

A finales de abril de 2009 la Organización Mundial de la Salud (OMS) calificó el brote por el nuevo virus de gripe $\mathrm{A}(\mathrm{H} 1 \mathrm{~N} 1)$, tras la notificación de los primeros casos en Estados Unidos ${ }^{4}$ y México ${ }^{5}$, como emergencia de Salud Pública de importancia internacional ${ }^{6}$. El 11 de junio elevó el nivel de alerta pandémica a fase 6 tras considerar la existencia de transmisión elevada y sostenida del virus en el mundo ${ }^{7}$. Al final del año 2009 la infección por el nuevo virus de la gripe $\mathrm{A}(\mathrm{H} 1 \mathrm{~N} 1)$ había sido detectada prácticamente en todos los países del mundo ${ }^{8}$.

Desde el primer momento en España se activó el Plan Nacional de Preparación y Respuesta ante una pandemia de gripe, coordinado por el Centro de Coordinación de Alertas y Emergencias Sanitarias (CCAES) del Ministerio de Sanidad y Política Social (MSPS), con la recomendación de reforzar y adaptar los sistemas de vigilancia existentes hasta entonces en las diferentes Comunidades Autónomas.
Coincidiendo estas fechas con el inicio de la temporada gripal en los países del hemisferio sur ${ }^{9}$, los primeros datos sobre la magnitud de la pandemia fueron obtenidos de Argentina ${ }^{10}$, Chile $^{11}$, Australia ${ }^{12}$, Nueva Zelanda ${ }^{13,14}$. Se observó una evolución de las primeras ondas pandémicas similares en duración a temporadas anteriores, dando lugar a un cuadro clínico de moderada gravedad con importante afectación de población infantil y adultos menores de 65 años. Además, el virus permaneció antigénicamente estable. Las tasas de ataque de enfermedad clínica en la semana del pico epidémico oscilaron en estos países entre $0,01 \mathrm{y}$ 0,03 casos por 100 habitantes. Mientras que la tasa de mortalidad ascendió a 1,25 por 100.000 habitantes en Argentina y entre 0,4 y 0,8 defunciones por 100.000 habitantes en el resto de los países.

En España las estrategias de vigilancia se fueron adaptando a la evolución de la pandemia, incluyendo modificaciones a lo largo de los meses. Durante las primeras semanas se realizó una vigilancia individual de los casos, con identificación y seguimiento de los contactos estrechos. A medida que el número de casos aumentaba la vigilancia se centró en los que requerían ingreso hospitalario por complicaciones de la infección. Además, el Sistema Centinela de Vigilancia de la Gripe en España (SVGE), que habitualmente permanece activo desde la semana 40 de un año hasta la 20 del siguiente, se prolongó durante el verano para monitorizar la actividad de la gripe pandémica en nuestro país.

En La Rioja, tras la notificación de alerta pandémica en abril de 2009, se activó el Plan de actuación frente a la pandemia de gripe en La Rioja ${ }^{15}$. Este plan responde a las recomendaciones emitidas desde la Organización Mundial de la Salud y la Unión Europea, bajo las directrices del Plan Nacional. Las actividades de vigilancia epidemiológi- 
ca se desarrollan a través del Subcomité de Vigilancia Epidemiológica constituido en el seno de dicho Plan.

Los objetivos de este estudio fueron realizar un análisis de la evolución de la pandemia por el virus de la gripe (H1N1) 2009 en la Comunidad Autónoma de La Rioja, tanto en el ámbito de la atención primaria como de las personas ingresadas con diagnóstico de gripe (H1N1) 2009.

\section{MATERIAL Y MÉTODOS}

El periodo de estudio incluye desde la semana epidemiológica 28-2009 (comienza el 18 de julio) hasta la semana 3-2010 (finaliza el 23 de enero).

\section{Casos sospechosos de gripe (H1N1) 2009 en Atención Primaria}

La información de los casos de gripe no ingresados se obtuvo semanalmente a partir de las bases informatizadas de atención primaria (OMI). Por lo que no se han incluido en este análisis aquellas zonas básicas de salud sin OMI (Cervera, Murillo, San Román y Torrecilla). La población cubierta por el sistema OMI asciende al 96,26\% del total de La Rioja.

\section{Casos de gripe (H1N1) 2009 hospitalizados}

La información de los casos sospechosos de gripe hospitalizados se obtuvo a partir de los listados diarios remitidos por los Servicios de Medicina Preventiva Hospitalarios y del laboratorio de referencia de La Rioja, cubriendo al $100 \%$ de la población de La Rioja.

En los casos ingresados en los que se con- firmó el diagnóstico de gripe (H1N1) 2009 por laboratorio, el médico responsable cumplimentó el formulario de notificación de casos graves de infección por virus pandémico, en el que se recogía información demográfica, factores de riesgo del paciente, datos de la evolución, complicaciones, tratamiento, vacunación y estatus final del paciente.

\section{Análisis de los datos}

Se realizó una descripción de los casos de gripe (H1N1) 2009 según su distribución por semana epidemiológica, sexo, grupo de edad, ámbito de atención (primaria/hospitalización), existencia de factores de riesgo (asma, EPOC, obesidad, diabetes, insuficiencia renal, inmunodeficiencia, cardiopatía, enfermedad hepática crónica, anemiahemoglobinopatía, enfermedad neuromuscular), evolución y complicaciones durante su enfermedad. Los resultados se expresaron como porcentaje en el caso de variables cualitativas y como media y desviación estándar en el de variables cuantitativas. En el caso de las mujeres embarazadas estos datos se calcularon sobre las mujeres de 15-49 años. La población pediátrica estaba constituida por los menores de 15 años.

Se calculó la proporción de pacientes que habían recibido tratamiento según grupo de edad, sexo, y factores de riesgo de complicaciones, así como la distribución del tiempo hasta el tratamiento y su duración: media (desviación estándar), mediana (rango intercuartílico). Se utilizó la regresión binaria logística para estimar las odds ratios ajustadas (OR ajustada) y su intervalo de confianza (IC) al 95\% de recibir tratamiento antiviral según factores de riesgo de riesgo. 


\section{RESULTADOS}

\section{Casos sospechosos de gripe (H1N1) 2009 en Atención Primaria en La Rioja (OMI-AP)}

El primer caso de infección por el virus pandémico confirmado por laboratorio en La Rioja se presentó durante la semana epidemiológica 27 de 2009 (del 5 al 11 de julio). Hasta la semana epidemiológica 3 de 2010, que finalizó el día 23 de enero, se declararon a través del sistema OMI de Atención Primaria 7.096 casos con sospecha de gripe (H1N1) 2009.

\section{Distribución en el tiempo}

A partir de la semana 28 de 2009 (12 al 18 de julio) la incidencia de casos de gripe (H1N1) 2009 permaneció por debajo del umbral basal establecido para el SVGE $(63,1$ casos por 100.000 habitantes), hasta la semana 42 (18 al 24 de octubre de 2009) en la que lo superó, alcanzando una incidencia de 97,8 casos por 100.000 habitantes. Desde entonces comenzó un ascenso que alcanzó el pico máximo en la semana 45 (369,6 casos/100.000 habitantes). Estas cifras se mantuvieron elevadas aunque en descenso hasta la semana 48 (29 de noviembre al 5 de diciembre), con una incidencia de 109,4 casos por 100.000 habitantes. Posteriormente las cifras presentaron una tendencia descendente y por debajo del umbral epidémico (figura 1).

\section{Características de las personas afectadas}

El 49,6\% fueron mujeres y el 50,3\% hombres. La edad media fue de 28,4 (DE: $\pm 18,7$ ) años, la mediana fue 26,3 (rango intercuartílico: 12,7-40,6) años, y la moda 37 años. Por edad se observó que los sujetos más afectados fueron los menores de 14 años, siendo el grupo que presentó más gripe el de 10-14 años (figura2).

\section{Factores de riesgo}

El 22\% ( $\mathrm{N}=1560)$ de los pacientes con sospecha de gripe (H1N1) 2009 en atención primaria presentó algún factor de riesgo. El $80,5 \%$ de los pacientes con factores de riesgo tenían uno y el máximo número de factores fue 6 en 2 personas $(0,13 \%)$.

El grupo de factores de riesgo más frecuente entre los diagnosticados de gripe (H1N1) 2009 fue el de las enfermedades respiratorias, seguido de las enfermedades metabólicas (obesidad y diabetes mellitus) (tabla 1).

\section{Figura 1}

Evolución de la incidencia de gripe (H1N1) 2009 en La Rioja

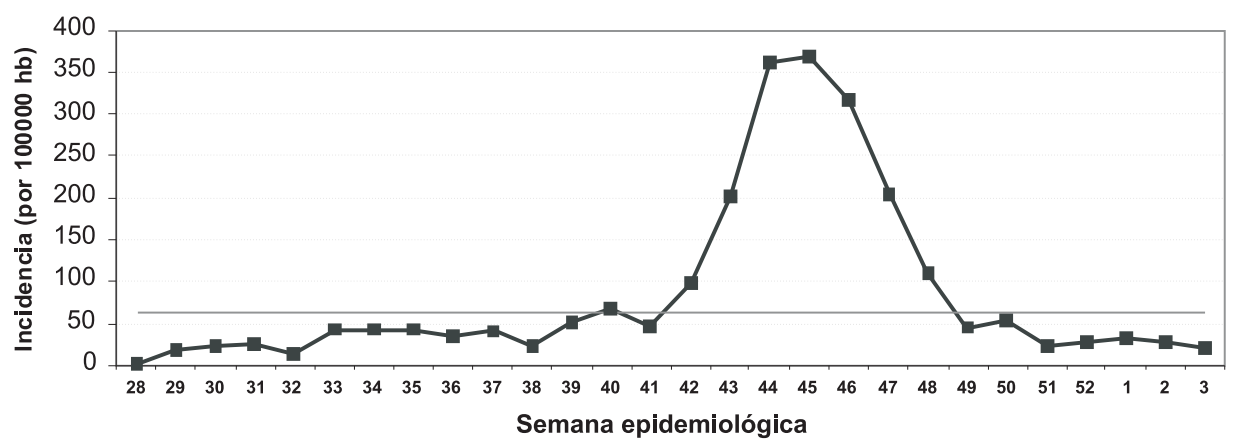


Figura 2

Incidencia por 100.000 habitantes y casos de gripe (H1N1) 2009 por grupos de edad en La Rioja

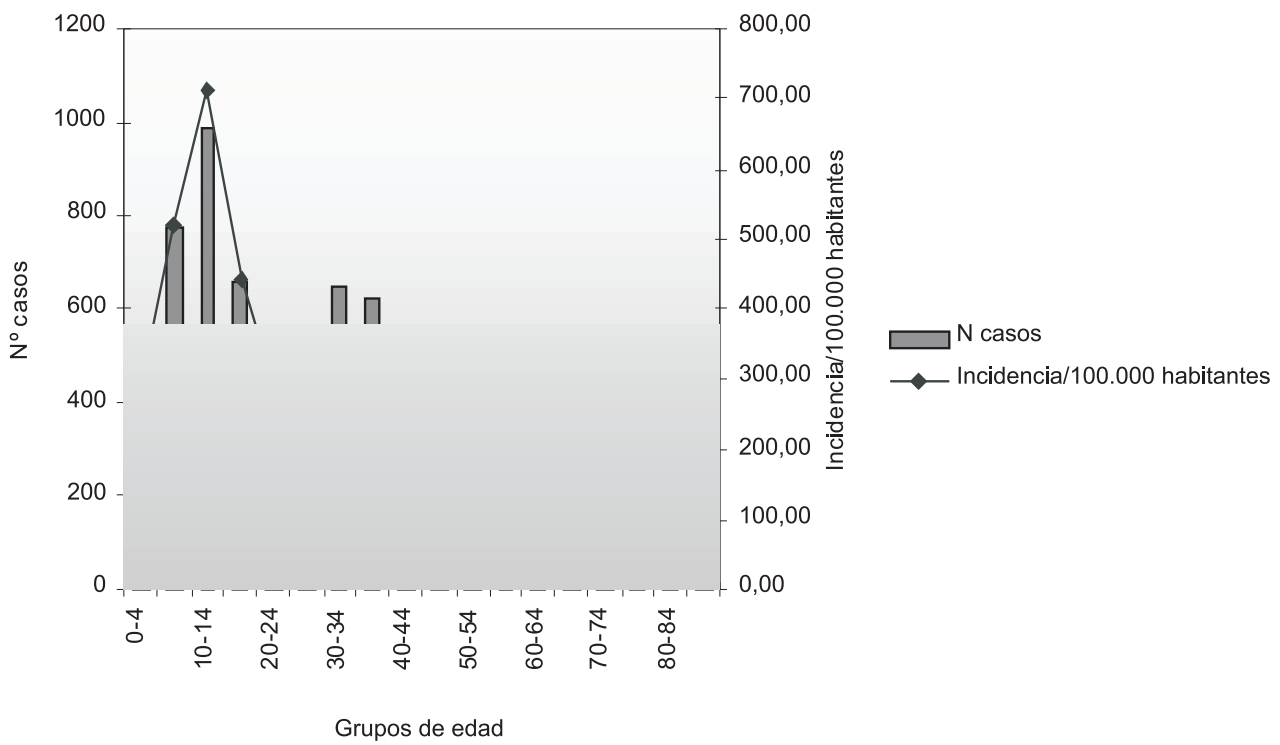

Tabla 1

Factores de riesgo en los pacientes con sospecha de gripe

\begin{tabular}{|l|r|}
\hline \multicolumn{1}{|c|}{ ENFERMEDAD (CIAP) } & \multicolumn{1}{|c|}{ N } \\
\hline Asma & 541 \\
EPOC & 52 \\
Otras enfermedades del aparato respiratorio & 112 \\
Anemia perniciosa-déficit folatos & 13 \\
Anomalías hemolíticas hereditarias & 12 \\
Otras anemias inespecíficas & 11 \\
Otras enfermedades hematológicas-inmunológicas-linfáticas & 3 \\
Otras anomalías congénitas de la sangre-hematológicas & 1 \\
Obesidad & 258 \\
Diabetes Mellitus & 193 \\
Enfermedades neurológicas & 151 \\
Isquemia cardiaca con angina & 20 \\
Insuficiencia Cardiaca & 21 \\
Enfermedades infeciosas cardiovasculares & 7 \\
Isquemia cardiaca sin angina & 3 \\
Arritmia cardiaca NE & 7 \\
Otras enfermedades cardiacas & 71 \\
Otras enfermedades cardiovasculares & 47 \\
Embarazo & 49 \\
Enfermedades Hepáticas no especificadas & 34 \\
Problemas-enfermedades urinarias & 32 \\
Infección por VIH-SIDA & 13 \\
Otras enfermedades generales no especificadas & 2 \\
\hline
\end{tabular}


Figura 3

Número de personas ingresadas por gripe (H1N1) 2009 y confirmadas en La Rioja por semana epidemiológica

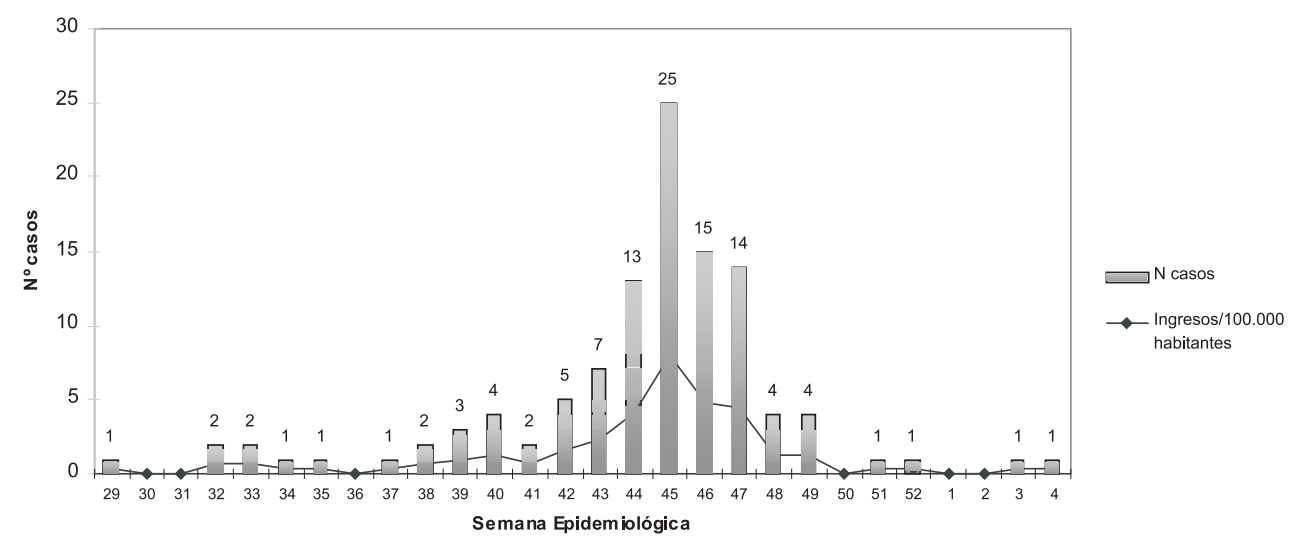

\section{Casos ingresados en La Rioja con diagnóstico confirmado por laboratorio de gripe (H1N1) 2009}

De los 111 sujetos hospitalizados con diagnóstico confirmado por laboratorio requirieron ingreso en cuidados intensivos 5 $(4,5 \%)$ y fallecieron $2(1,8 \%)$.

\section{Distribución en el tiempo}

En la distribución por semana epidemiológica se observó que el mayor número de ingresos se produjo entre las semanas 44 y 47 , alcanzándose el pico máximo la semana 45 (del 8 al 14 de noviembre de 2009) con 25 personas hospitalizadas y una incidencia de 8,1 ingresos por 100.000 habitantes (figura 3).

\section{Características de las personas ingresadas}

El 50,9\% de las personas ingresadas con gripe (H1N1) 2009 fueron hombres frente al $49,1 \%$ que fueron mujeres. La edad media de los pacientes ingresados y con confirmación diagnóstica por laboratorio fue de 34,5(DE: $\pm 22,6$ ) años, la mediana 32 años (rango intercuartílico: 15,7-50,2).
Por grupos de edad se observó que los ingresaron con mayor frecuencia fueron los menores de cinco años seguidos de los grupos de edad de 10-14 años y de 25-29 años (figura 4).

Los 27 enfermos pediátricos ( $<15$ años) supusieron un $24,3 \%$ de los casos ingresados. Ninguno de ellos requirió ingreso en cuidados intensivos. Los sujetos menores de 2 años supusieron el 10,9\% del total de los hospitalizados y el $44,4 \%$ de los pediátricos.

De las 30 mujeres en edad fértil (15-49 años), 10 estaban embarazadas (33,3\%), 2 de ellas en el primer trimestre, 2 en el segundo y 6 en el tercero.

La edad media de los 5 sujetos ( 3 hombres y 2 mujeres) que requirieron ingreso en la Unidad de Cuidados Intensivos (UCI) fue de 41,8 (DE: $\pm 14,7)$ años, con un rango de 2664 años.

\section{Factores de riesgo en las personas ingresadas}

En 78 (70,9\%) de los sujetos ingresados se recogió la presencia de algún factor de 


\section{Figura 4}

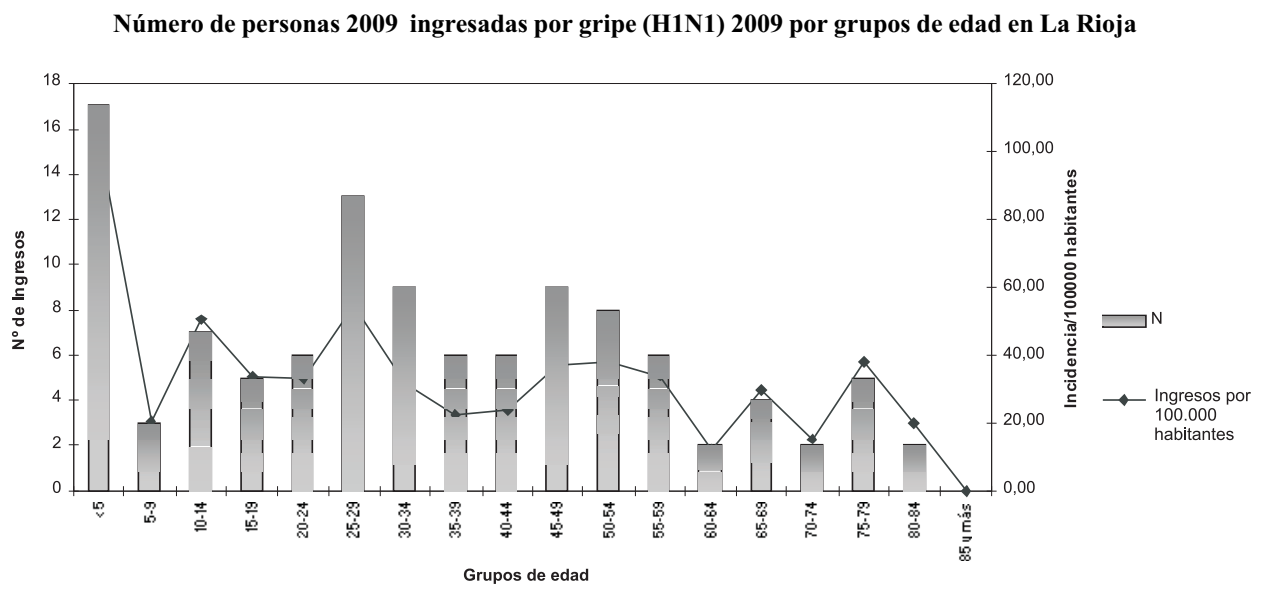

Figura 5

Presencia de factores de riesgo en personas ingresadas por gripe (H1N1) 2009*

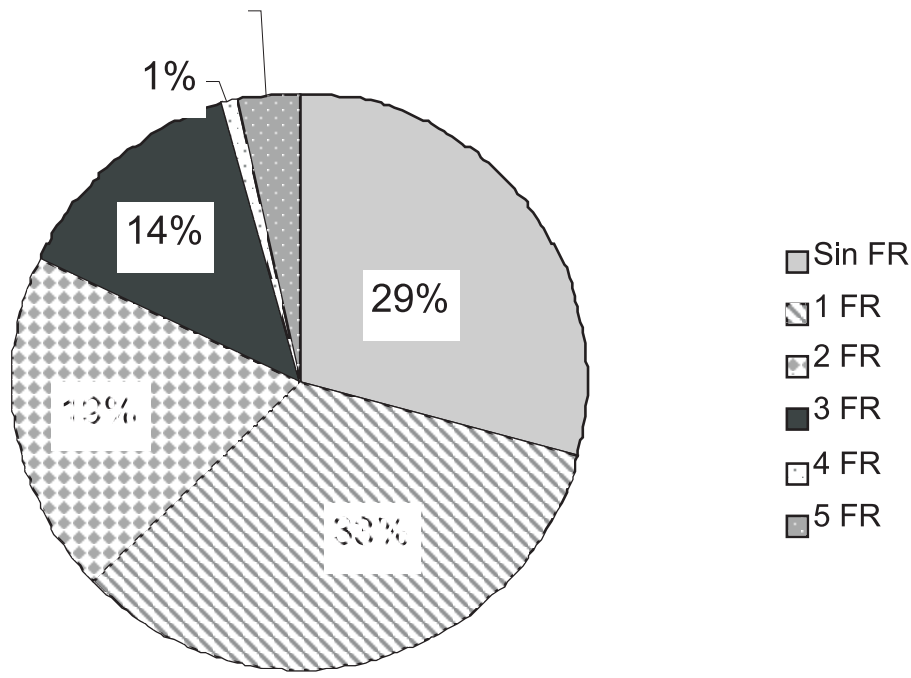

riesgo. $37(36,6 \%)$ presentaron un único factor de riesgo, $4(3,6 \%)$ que presentaron 5 factores (figura 5).

Los factores de riesgo que se presentaron de forma más frecuente fueron las enfermedades pulmonares, que aparecieron en 31
(28,1\%) sujetos ingresados: 9 asma, 10 EPOC y 12 otras enfermedades), y el hábito de consumo de tabaco que se dio en 21 $(19,8 \%)$ personas (tabla 2).

En 4 pacientes que requirieron ingreso en UCI las enfermedades respiratorias constitu- 
Tabla 2

Factores de riesgo al ingreso

\begin{tabular}{|l|r|r|}
\hline \multicolumn{1}{|c|}{ FACTORES DE RIESGO } & n/Total & \multicolumn{1}{c|}{$\%$} \\
\hline Fumador actual & $21 / 106$ & $19,81 \%$ \\
Asma & $9 / 103$ & $8,74 \%$ \\
EPOC & $10 / 107$ & $9,35 \%$ \\
Otra enfermedad pulmonar crónica & $12 / 106$ & $11,32 \%$ \\
Obesidad IMC $>40$ & $7 / 109$ & $6,42 \%$ \\
Diabetes & $11 / 109$ & $10,09 \%$ \\
Otros desórdenes metabólicos & $9 / 106$ & $8,49 \%$ \\
Insuficiencia Renal & $5 / 108$ & $4,63 \%$ \\
Cáncer & $14 / 110$ & $12,73 \%$ \\
Inmunodeficiencias & $15 / 106$ & $14,15 \%$ \\
Cardiopatía & $10 / 107$ & $9,35 \%$ \\
Enfermedad Hepática Crónica & $5 / 102$ & $4,90 \%$ \\
Anemia-hemoglobinopatía & $10 / 110$ & $9,09 \%$ \\
Transtornos Convulsivos & $1 / 106$ & $0,94 \%$ \\
Disfunción Cognitiva & $8 / 104$ & $7,69 \%$ \\
Asplenia & $2 / 108$ & $1,85 \%$ \\
Enfermedad Neuromuscular & $2 / 107$ & $1,87 \%$ \\
Tratamiento crónico con AAS & $4 / 98$ & $4,08 \%$ \\
Embarazo* & $10 / 30$ & $66,67 \%$ \\
\hline
\end{tabular}

* Datos calculados sobre la población de mujeres en edad fértil (15-49 años).

yeron el principal factor de riesgo (2 síndrome de apnea obstructiva del sueño (SAOS), EPOC en 1 caso y asma en otro, en segundo lugar el consumo de tabaco $(n=3)$ y la obesidad estaba presente en 2 pacientes, ocupando el tercer lugar. Otros factores presentes fueron diabetes (1), cardiopatía (1), insuficiencia renal (1), inmunodeficiencia (1) y enfermedad hepática crónica (1).

Los factores de riesgo de los dos pacientes fallecidos fueron en el primer caso SAOS, diabetes, dislipemia, enfermedad cardiovascular, tratamiento prolongado con ácido acetil salicílico. Y en el segundo caso: asma, EPOC, SAOS, obesidad mórbida, tabaquismo.

\section{Evolución clínica y complicaciones}

La forma de presentación de la enfermedad fue un cuadro gripal en $98(89,9 \%)$ per- sonas, y en forma de neumonía en 10 $(9,1 \%)$.

El 47,2\% (52) presentaron algún tipo de complicación durante la evolución del cuadro gripal. La complicación más frecuente fue la neumonía $(37,2 \%)$ seguida de hipoxemia que apareció en el $22,4 \%$ de los casos (tabla 3).

En los pacientes ingresados en UCI las complicaciones más frecuentes fueron también la neumonía y la hipoxemia. Dos de estos pacientes fallecieron durante su estancia en dicha unidad, presentando ambos como complicaciones el SDRA, sepsis, shock y fracaso multiorgánico (tabla 4).

Dos de los pacientes ingresados en UCI fueron los fallecidos en La Rioja, dando lugar a una tasa de mortalidad de 0,62/ 100.000 habitantes. Ambos pacientes preci- 
Tabla 3

Complicaciones durante la estancia en el hospital

\begin{tabular}{|l|r|r|}
\hline \multicolumn{1}{|c|}{ COMPLICACIONES TOTAL } & n/Total & \% \\
\hline Neumonía & $41 / 110$ & $37,27 \%$ \\
Hipoxemia & $24 / 107$ & $22,43 \%$ \\
SDRA* & $2 / 110$ & $1,82 \%$ \\
CID** & $1 / 110$ & $0,91 \%$ \\
Sepsis & $2 / 107$ & $1,87 \%$ \\
Shock & $2 / 110$ & $1,82 \%$ \\
Fracaso Multiorgánico & $2 / 110$ & $1,82 \%$ \\
Alteración Cardiaca & $2 / 110$ & $1,82 \%$ \\
Alteración de la función hepática & $1 / 109$ & $0,92 \%$ \\
Fracaso Renal Agudo & $2 / 109$ & $1,83 \%$ \\
\hline
\end{tabular}

* Síndrome de Distress Respiratorio del Adulto.

** Coagulación Intravascular Diseminada.

Tabla 4

Complicaciones durante la estancia en UCI

\begin{tabular}{|l|c|}
\hline \multicolumn{1}{|c|}{ COMPLICACIONES } & n (TOTAL = 5) \\
\hline Neumonía & 4 \\
Hipoxemia & 5 \\
SDRA & 2 \\
CID & 1 \\
Sepsis & 2 \\
Shock & 2 \\
Fracaso Multiorgánico & 2 \\
Alteración Cardiaca & 1 \\
Alteración de la función hepática & 1 \\
Fracaso Renal Agudo & 1 \\
\hline
\end{tabular}

saron ventilación mecánica y uno de ellos diálisis. Otro de los casos con estancia en UCI recibió también ventilación mecánica.

\section{Tratamiento antiviral}

Del total de pacientes ingresados recibieron tratamiento antiviral el 73,6\% (81). La duración del tratamiento fue de 5 días en el $95,1 \%(77)$.

Los pacientes pediátricos ( $<15$ años $)$ constituyeron el grupo en el que con menor fre- cuencia se prescribió oseltamivir. El 81,4\% de los niños no recibieron tratamiento antiviral durante su ingreso, frente al 18,5\% a los que sí se les había prescrito.

El grupo de edad de 15-64 años fue tratado con antivirales en el $92,8 \%$, y el de los mayores de 64 en un $84,6 \%$ de las ocasiones. De las 10 mujeres embarazadas 7 fueron tratadas con oseltamivir.

El tiempo desde el inicio de los síntomas hasta que los pacientes recibieron tratamiento presentó una media de 3,1 (DE: 3,00) días. 
Tabla 5

Mediana y rango intercuatílico de la evolución temporal del cuadro clínico en las personas ingresadas

\begin{tabular}{|l|c|c|c|c|}
\hline & $\mathbf{0 - 1 4}$ años & $\mathbf{1 5 - 6 5}$ años & $>\mathbf{6 5}$ años & TOTAL \\
\hline $\begin{array}{l}\text { Días desde el inicio de síntomas hasta } \\
\text { ingreso hospitalario }\end{array}$ & $2(0-5)$ & $2(1-4)$ & $2(1,5-4)$ & $2(1-4)$ \\
\hline $\begin{array}{l}\text { Días desde inicio de síntomas hasta } \\
\text { inicio de tratamiento }\end{array}$ & $2(1-3,5)$ & $3(1-4)$ & $3(3-7)$ & $3(1-4)$ \\
\hline Días de enfermedad & $6(4-10)$ & $7,5(6-10)$ & $10(8-11)$ & $8(5,7-10)$ \\
\hline Días de ingreso & $3(2-5)$ & $5(4-6)$ & $6(6-9)$ & $5(3-6)$ \\
\hline
\end{tabular}

La mediana fue 3 (rango 1-4) días. El mínimo fue 0 días (comienzo de tratamiento el mismo día de inicio de síntomas) y el máximo de 10 .

El 44,4\% (36) de los pacientes recibió el tratamiento antiviral durante las primeras 48 horas desde el inicio de los síntomas.

El 83\% de los que presentaban factores de riesgo frente al $50 \%$ sin estos factores, recibieron tratamiento antiviral (OR ajusta$\mathrm{da}=2,17$; IC95\% 0,75-6,24). El 73\% de los que debutaron con un cuadro gripal y el $80 \%$ de los que tenían neumonía recibieron tratamiento (OR ajustada=1,69; IC95\% 0,2710,38). El asma (OR ajustada $=3,28 ; \mathrm{IC} 95 \%$ $0,38-31$ ) y EPOC (OR ajustada=2,57; IC95\%0,22-21,49) fueron los factores en los que más aumentó la administración del tratamiento. La insuficiencia renal se asoció significativamente a una menor frecuencia de administración de antiviral (OR ajusta$\mathrm{da}=0,07$; IC95\%0,006-0,846).

\section{Evolución temporal de la estancia hospitalaria}

La estancia de los pacientes en el hospital, así como los días de duración de la enfermedad, aumentó a medida que aumentaba su edad, existiendo diferencias estadísticamen- te significativas entre el grupo de menor y mayor edad (tabla 5).

La estancia de los 5 pacientes que ingresaron en UCI, presentó una mediana de 4 días (rango intercuartílico: 1-15,5). La media fue 7,4 (DE: 10,1) días. La estancia mínima fue 1 día y la máxima 25 .

\section{DISCUSIÓN}

Desde la aparición del primer caso confirmado de gripe (H1N1) 2009 en España, el día 26 de abril, hasta que se confirmó en La Rioja un caso el día 11 de julio de 2009 y a pesar de intensificar las medidas de vigilancia en todos los casos sospechosos, transcurrieron 2 meses y medio, siendo una de las Comunidades Autónomas en la que la gripe (H1N1) 2009 apareció de forma más tardía, posiblemente por el tamaño de la población de La Rioja.

En cuanto a la distribución temporal de casos de gripe durante la pandemia, se observa que, como era de esperar, la situación que ocurre a nivel comunitario, queda reflejada en la distribución de los ingresos hospitalarios, presentando una curva similar en el tiempo ambas situaciones.

A pesar de que la gripe fue más frecuente en los grupos de edad más jóvenes - al igual 
que lo que había sucedido en los países del hemisferio sur ${ }^{9}$ - se observó que la edad de los pacientes ingresados era mayor que la de los casos diagnosticados y manejados de forma ambulatoria. El aumento de hospitalizaciones en las personas mayores puede estar relacionado con el mayor número de factores de riesgo y comorbilidades que presentan las personas que pertenecen a estos grupos de edad, que a su vez hace que sean los que tienen una estancia más larga durante su ingreso hospitalario.

En cuanto al cuadro clínico de la enfermedad en la población riojana, evolucionó de forma favorable en la mayoría de los casos diagnosticados. Esta situación ya se había observado en los países del hemisferio sur, en los que se presentó un cuadro clínico de moderada gravedad ${ }^{9}$.

Aunque la tasa de ataque de enfermedad clínica en el pico de la pandemia fue en torno a 10 veces más elevada en la población de La Rioja que en los países del hemisferio sur $^{9-14}$, en comparación con estos países, la proporción de casos clínicos de gripe que fueron hospitalizados durante el periodo pandémico en nuestra comunidad, fue similar, aunque ligeramente superior a la presentada en Chile ${ }^{11}$ y en Argentina ${ }^{10}$, mientras que es mucho menor a la que se observó en Nueva Zelanda ${ }^{13,14}$. La tasa de letalidad fue menor que la registrada en cualquiera de los países del hemisferio sur de los que disponemos de datos.

La presencia de factores de riesgo de complicaciones por gripe (H1N1) 2009 fue significativamente mayor en los pacientes hospitalizados, que en los que fueron manejados desde Atención Primaria. Sin embargo, desconocemos si la existencia de estos factores supuso un motivo de ingreso, para evitar que aparezcan otras complicaciones durante la enfermedad, intensificando la vigilancia de estos pacientes.

Las recomendaciones de los protocolos de referencia ${ }^{15}$, respecto al tratamiento antivi- ral, eran en primer lugar la administración del mismo en todos los pacientes ingresados a causa de la gripe (H1N1). En nuestro estudio, se ha observado que no se siguió esta recomendación en el total de los casos hospitalizados, aunque se administró el antiviral a las tres cuartas partes de los pacientes. La segunda recomendación era la administración del mismo en las primeras 48 horas tras el diagnóstico, que en nuestro caso se cumplió en menos de la mitad de los infectados, si bien habría que tener en cuenta en este bajo porcentaje de cumplimento, la posibilidad de que los pacientes fueran ingresados con más de dos días de evolución de su enfermedad, llegando al diagnóstico de gripe después de dicho periodo. Además, los menores de 15 años que requirieron ingreso, constituyen el grupo de edad en el que menos se ha pautado el tratamiento antiviral, a pesar de estar indicado, sin embargo todos ellos han presentado una evolución favorable. El motivo de la baja prescripción del antiviral en esta población pediátrica puede estar relacionado con la precaución de administración de medicación en los niños, teniendo en cuenta la valoración de riesgo y beneficio por parte del médico encargado del caso, así como por la limitada experiencia de uso en los niños menores de 1 año.

Como limitación principal de este estudio, se encuentra que el diagnóstico de gripe (H1N1) en los pacientes ambulatorios cuya evolución se siguió desde Atención Primaria, está basado en una sospecha clínica en la gran mayoría de los casos, sin haberse realizado una confirmación microbiológica (no así en los casos ingresados, en los que a través de frotis nasofaríngeo se identificó el virus en todos los casos). Está situación puede dar lugar a una sobreestimación del número de casos de gripe manejados desde Atención Primaria, teniendo en cuenta además que nos encontrábamos en una situación de alerta de pandemia, durante la cual la sospecha de gripe en la población podía ser mayor que en cualquier otra temporada. 
Como conclusiones, se puede destacar que la gripe (H1N1) 2009 en La Rioja ha presentado una evolución clínica favorable en la inmensa mayoría de los afectados, presentando peor evolución aquellas personas con factores de riesgo. Además, al igual que en otros países del hemisferio sur, ha afectado principalmente a la población joven, siendo menos frecuente la aparición en personas mayores de 60 años.

\section{BIBLIOGRAFÍA}

1. Kilbourne ED. Influenza pandemics of the 20th Century. Emerg Infect Dis. 2006; 12: 9-14.

2. Taubenberger JK, Morens DM. 1918 Influenza: the mother of all pandemics. Emerg Infect Dis. 2006; 12(1): $15-22$.

3. Viboud C. Mutinational impact of the 1968 Hong Kong influenza pandemic: evidence for a smouldering pandemic. J Infect Dis. 2005; 192(2): 233-48.

4. Centers for Disease Control and Prevention (CDC). Swine Influenza A(H1N1) infections California and Texas, April 2009. MMWR Morb Mortal Wkly Rep. 2009;58:(16):435-7. Disponible en: http://www.cdc.gov/mmwr/preview/mmwrhtml/m m5816a7.htm.

5. Centers for Disease Control and Prevention (CDC). Outbreak of swine-origin influenza A(H1N1) virus infection-Mexico, March-April 2009. MMWR Morb Mortal Wkly Rep. 2009;58:467-470 Disponible en: www.cdc.gov/mmwr/preview $/ \mathrm{mm}$ $\mathrm{wrhtml} / \mathrm{mm} 58 \mathrm{~d} 0430 \mathrm{a} 2 . \mathrm{htm}$.

6. WHO. Statement to the press by WHO DirectorGeneral Dr Margaret Chan, 25 April 2009. Swine influenza. Disponible en: http://www.who.int/ mediacentre/news/statements/2009/h1n1_200904 25/en/index.html.

7. WHO. Statement to the press by WHO DirectorGeneral Dr Margaret Chan, 11 June 2009. World now at the start of 2009 influenza pandemic. Disponible en: http://www.who.int/mediacentre/ news/statements/2009/h1n1_pandemic_phase6_20090611/en/index.html.

8. WHO. Pandemic (H1N1) 2009 - update 78 (11 December 2009). Disponible en: http://www. who.int/csr/don/2009 12 11a/en/index.html.

9. Department of Health and Human Services. Assesment of the 2009 Influenza A (H1N1) Pandemic on selected countries in the Southern hemisphere: Argentina, Australia, Chile, New Zealand and Uruguay. White House National Security Council. August 26, 2009. Disponible en: http://www. flu.gov/professional/global/final.pdf.

10. Página oficial del Ministerio de Salud de Argentina, sobre la pandemia de influenza. Influenza pandémica (H1N1) 12-09-2009. Disponible en: http:// www.msal.gov.ar/archivos/12-09-09\%20INFORME\%20INFLUENZA\%20PANDEMICA\%20 H1 N1_.pdf.

11. Chile. Ministerio de Salud. Informe de prensa 16 de septiembre de 2009. Disponible en: http://www. pandemia.cl/pagnew/prensa/REPORTE_16_09 09.pdf.

12. Department of Health and Ageing. Australian Government. Disponible en: http://www.healthemergency.gov.au/internet/healthemergency/publishing.nsf/Content/ozflu2009.htm.

13. Nueva Zelanda. Public Health Surveillance. Disponible en: http://www.surv.esr.cri.nz/virology/influenza_weekly_update.php.

14. Public Health Surveillance. Information for New Zealand Public Health Action. Virological Surveillance. Influenza weekly update. Disponible en: http:/www.surv.esr.cri.nz/virology/influenza_wee kly_update.php.

15. Dirección General de Salud Pública y Consumo. Plan de preparación y respuesta frente a la pandemia de gripe en La Rioja. Logroño: Consejería de Salud de La Rioja; 2009. 